\title{
Influence of Host Resistance and Insecticide Seed Treatments on Curly Top in Sugar Beets
}

Carl A. Strausbaugh and Anne M. Gillen, USDA-ARS NWISRL, 3793 North 3600 East, Kimberly, ID 83341; John J. Gallian, University of Idaho, Research and Extension Center, Twin Falls, ID 83303; Stacey Camp, Amalgamated Sugar Co., 50 S. 500 W., Paul, ID 83347; and J. R. Stander, Betaseed Inc., P.O. Box 895, Kimberly, ID 83341

\begin{abstract}
Strausbaugh, C. A., Gillen, A. M., Gallian, J. J., Camp, S., and Stander, J. R. 2006. Influence of host resistance and insecticide seed treatments on curly top in sugar beets. Plant Dis. 90:15391544.

Curly top on sugar beets (Beta vulgaris) caused by Beet severe curly top virus or closely related species is a considerable problem in arid growing regions of the western United States. Two insecticide seed treatments, Poncho Beta $(60 \mathrm{~g}$ a.i. clothianidin $+8 \mathrm{~g}$ a.i. beta-cyfluthrin $/ 100,000$ seed) and Gaucho (45 g a.i. imidacloprid/100,000 seed), and four sugar beet hybrids varying in curly top resistance were evaluated for their influence on the control of curly top in comparison with untreated checks. Plots were established at two locations in southern Idaho in 2005 and evaluated for curly top. Moderate to severe curly top due to natural inoculum and leafhopper infestations occurred at both locations. Untreated, the four hybrids performed as expected with the fewest curly top symptoms on PM21 and the most on Monohikari. Both insecticide treatments lowered curly top ratings compared with the untreated check, but Poncho Beta reduced symptoms more than Gaucho as the season progressed. Poncho Beta led to increased yield and estimated recoverable sugar across all hybrids at harvest, particularly on the more susceptible hybrids. When considering the yield parameters for only the most resistant hybrids individually, Poncho Beta did not always outperform Gaucho. Poncho Beta provided a level of control that would justify its application as a supplement to host resistance under Idaho conditions.
\end{abstract}

Additional keywords: Curtovirus, geminivirus

Curly top on sugar beets (Beta vulgaris) is a problem in arid growing regions of the western United States (2). Curly top can infect over 300 plant species and cause disease on a number of important crops such as sugar beet, tomato, bean, pepper, cucumber, melon, spinach, and squash $(1,2,5,6)$. Curly top on sugar beets is caused by Beet severe curly top virus (BSCTV) or a number of other closely related species transmitted by the beet leafhopper, Circulifer tenellus (Baker), in a circulative-nonpropagative manner $(16,17)$. In southern Idaho, a limited survey indicated that BSCTV, previously called Beet curly top virus strain $\mathrm{CFH}$, was the primary species present (18).

Curly top nearly eliminated the sugar beet industry in southern Idaho until beets with resistance became generally available in 1935 (2,3). Since that time, host resistance has been the primary means of con-

Corresponding author: C. A. Strausbaugh

E-mail: carls@nwisrl.ars.usda.gov

Accepted for publication 12 August 2006.

DOI: 10.1094/PD-90-1539

This article is in the public domain and not copyrightable. It may be freely reprinted with customary crediting of the source. The American Phytopathological Society, 2006. trol for curly top in sugar beets. However, even the most resistant commercial and experimental hybrids rated 2.5 to 3.0 on a disease index from 0 (healthy) to 9 (dead) (11), while the average rating for commercial hybrids under natural conditions in the 2004 variety trial in Nampa, ID, was 4.1 $(4,19)$. Early planting when possible can reduce disease severity, but is not always successful because of the unpredictable incidence and movement of viruliferous leafhoppers (22). Insecticidal sprays have not always provided effective control, while some soil-applied systemic insecticides have been more promising $(2,3)$. Phorate (Thimet) and aldicarb (Temik) are two systemic insecticides approved for use on sugar beets, but they are primarily targeted at root maggot control (3). These soil-applied insecticides have been somewhat effective in suppressing curly top (3). However, they are considered environmentally undesirable, and more effective control measures are needed in this regard.

Resistance to curly top is quantitatively inherited, which makes combining this resistance with resistance to other diseases difficult. In the early 1990s, rhizomania, caused by Beet necrotic yellow vein virus (BNYVV) and vectored by the plasmodiophorid Polymyxa betae Keskin, became an important widespread problem. BNYVV prevents normal root growth and causes severe yield loss, making rhizomania one of the most destructive diseases of sugar beet. Therefore, seed companies have rapidly incorporated resistance to BNYVV into commercial hybrids but found maintaining curly top resistance and high yield difficult in hybrids developed for the $\mathrm{Pa}-$ cific Northwest. Cultivars with resistance to rhizomania developed in the 1990s are just now approaching desired levels of curly top resistance (4). Unfortunately, $R z 1$, the single dominant gene for resistance to rhizomania, which is currently the most widely used source, appears to be compromised by new strains of BNYVV (9). Thus, additional sources of resistance to BNYVV will need to be incorporated into commercial cultivars. However, as additional rhizomania resistance is incorporated and becomes available, it will take time to bring these cultivars up to acceptable levels of curly top resistance. Transgenic cultivars with resistance to glyphosate are on the horizon. Breeders will also likely struggle to maintain acceptable levels of curly top resistance in these transgenic cultivars as they are developed. Therefore, managing curly top on sugar beets will continue to be both multifaceted and difficult. An important component of this management strategy will need to be the application of insecticides. In an effort to develop improved and more environmentally friendly control measures for curly top, we conducted studies to evaluate the relative influence of host resistance and insecticide seed treatments on the control of curly top in sugar beets.

\section{MATERIALS AND METHODS}

Treatments. Three seed treatments and four sugar beet hybrids with different levels of host resistance (4), HM PM21 = high, Beta $8600=$ high-intermediate, Phoenix R = low-intermediate, and Monohikari = highly susceptible, were evaluated. The insecticide seed treatments included an untreated check (no insecticide), Gaucho (45 g a.i. imidicloprid/100,000 seed), and Poncho Beta $(60 \mathrm{~g}$ a.i. clothianidin $+8 \mathrm{~g}$ a.i. beta-cyfluthrin/ 100,000 seed). In addition to the insecticide treatments, all seed were treated with the fungicides Allegiance FL (15.6 g a.i. metalaxyl $/ 100 \mathrm{~kg}$ seed) and Thiram $42 \mathrm{~S}$ ( $250 \mathrm{~g}$ a.i. thiram $/ 100 \mathrm{~kg}$ seed) to limit the influence of fungal pathogens and allow 
for good stand establishment. The seed treatments were applied to large raw conditioned seed by Gustafson Seed Technology Center, McKinney, TX. With 4 cultivars and 3 insecticide seed treatments, there were a total of 12 treatments. The experimental design was a randomized complete block design with eight replications. The experiment was conducted in Twin Falls County near Kimberly, ID, and repeated $232 \mathrm{~km}$ away in Ada County near Nampa, ID, in an area that historically (3) has more severe curly top than the Kimberly area.

Kimberly trial. The Kimberly trial was planted on the USDA-ARS Research Farm near Kimberly at 1,200 m elevation in an area considered to have moderate curly top disease pressure (3). The trial relied on the natural leafhopper population and virus for infection. The field had been in barley in 2004 and was plowed on 7 March 2005. Fertilizer (112 kg N/ha and $134 \mathrm{~kg}$ $\mathrm{P}_{2} \mathrm{O}_{5} /$ ha) was applied on 7 April and incor- porated with a roller harrow. The fumigant Telone II (94\% 1,3-dichloropropene) at 187.1 liters/ha was applied on 15 April to control unwanted soilborne influences from fungi and insects and then roller harrowed. The herbicide Ro-Neet 6E $(73.9 \%$ $s$-ethyl cyclohexylethylthiocarbamate) at 4.68 liters/ha was applied on 4 May and roller harrowed twice. The field trial was planted on 6 May 2005. The plots were planted to a density of 350,882 seeds/ha, and thinned to 88,066 plants/ha. Plots were four rows wide (56-cm row spacing) and $10.7 \mathrm{~m}$ long. The herbicides Progress $(7 \%$ phenmedipham $+7 \%$ desmedipham $+7 \%$ ethofumesate) at 1.53 liters/ha and Upbeet (50\% triflusulfuron methyl) at $35 \mathrm{~g} / \mathrm{ha}$ were applied together in 117.9 liters water/ha on 25 May. The field was rolled with a cultipacker to break up soil crust on 26 May. The crop was managed using standard cultural practices. Irrigation water was applied through handlines as needed. Prior to thinning, a stand count was taken

Table 1. Beet curly top disease rating system utilized by the Beet Sugar Development Foundation

\begin{tabular}{cl}
\hline Rating & Description of plant symptoms ${ }^{\mathbf{z}}$ \\
\hline 0 & Healthy; no symptoms \\
1 & Vein clearing of heart leaves, slight pimpling of veins on underside of leaves \\
2 & $\begin{array}{l}\text { Slight leaf curl of the edges of new leaves; pimpling on veins of underside } \\
\text { of leaves }\end{array}$ \\
3 & Center few whorls of leaves with curling edges \\
4 & Most leaves moderately curling; more than half of upper surface of leaf visible \\
5 & Slight stunting, severe leaf curling; less than half of upper leaf surface visible due \\
6 & to curling; most larger leaves still erect \\
7 & Stunting, slight yellowing; most leaves becoming prostrate \\
8 & Severe stunting, yellowing; leaves prostrate and some leaves dead \\
9 & Only the center few whorls of leaves green and alive \\
\hline
\end{tabular}

$\overline{{ }^{\mathrm{z}} \text { Rating system was published by David Mumford in } 1974 \text { (11). We utilized the rating system in a }}$ continuous manner rather than categorically. Thus, any number, including decimal numbers, between zero and nine was possible when scoring plants.

on 19 May when the plants had only cotyledons and no true leaves. The number of plants with spinach leafminer (Pegomya hyoscyami) in the two center rows was recorded on 21 June at the eight-leaf growth stage. Disease data were recorded for the center two rows on $13 \mathrm{July}, 9 \mathrm{Au}$ gust, and 7 September by giving the center two rows of the plot an overall severity rating using a disease index of 0 to 9 (Table 1). The field was sprayed with the fungicides Headline (23.6\% pyraclostrobin) at $841 \mathrm{~g} / \mathrm{ha}$ and sulfur at $6.72 \mathrm{~kg} / \mathrm{ha}$ on 13 August for control of powdery mildew. The center two rows were harvested on 27 October using a small plot harvester. During harvest, two eight-beet sugar samples per plot were collected for sugar analysis.

Nampa trial. The Nampa trial was conducted in a commercial sprinkler-irrigated sugar beet field near Nampa (approximately $16 \mathrm{~km}$ from the site of the 2004 variety trial [4,19] at Nampa) at $800 \mathrm{~m}$ elevation in an area considered to have severe curly top disease pressure. The field trial relied on the natural leafhopper population and virus for infection. The field had been planted to wheat in 2004. The field was fall plowed, land planed, and fall bedded with $22.4 \mathrm{~kg} \mathrm{~N}, 44.8 \mathrm{~kg} \mathrm{P}_{2} \mathrm{O}_{5}, 31.4 \mathrm{~kg}$ $\mathrm{K}, 43.7 \mathrm{~kg} \mathrm{~S}, 1.17$ liters $\mathrm{Zn}, 4.68$ liters boron, and 3.8 liters humic acid per ha. The field was also row fumigated in the fall with $85 \mathrm{ml}$ K-PAM HL (54\% potassium $N$-methyldithiocarbamate) per 30.48 $\mathrm{m}$ row. In spring, the herbicide Nortron SC (42\% ethofumesate) was broadcast at 2.33 liters/ha and mechanically worked into the beds prior to planting. The plots were planted on 24 March 2005 to a density of 469,490 seeds/ha, and thinned to 88,066 plants/ha on 10 May. Plots were four rows

Table 2. Influence of sugar beet host resistance and insecticide seed treatments on curly top during the 2005 growing season in Kimberly, ID

\begin{tabular}{|c|c|c|c|c|c|c|c|c|c|}
\hline \multirow{2}{*}{$\begin{array}{l}\text { Sugar beet } \\
\text { hybridu }^{u}\end{array}$} & \multirow{2}{*}{$\begin{array}{l}\text { Insecticide seed } \\
\text { treatment }^{v}\end{array}$} & \multirow{2}{*}{$\begin{array}{l}\text { No. plants } \\
\text { per } 3 \mathbf{~ m}^{\mathbf{w}}\end{array}$} & \multirow{2}{*}{$\begin{array}{c}\text { Plants }^{x} \\
\text { with } \\
\text { leafminer } \\
(\%)\end{array}$} & \multicolumn{3}{|c|}{ Curly top rating ${ }^{y}$} & \multirow{2}{*}{$\begin{array}{l}\text { Root } \\
\text { yield } \\
\text { (t/ha) }\end{array}$} & \multirow{2}{*}{$\begin{array}{c}\text { Sugar } \\
\text { content } \\
(\%)\end{array}$} & \multirow{2}{*}{$\begin{array}{c}\text { Estimated } \\
\text { recoverable } \\
\text { sugar } \\
(\mathrm{kg} / \mathrm{ha})\end{array}$} \\
\hline & & & & 13 Jul & 9 Aug & $7 \mathrm{Sep}$ & & & \\
\hline HM PM21 & Untreated check & $39.2 \mathrm{bc}$ & $82.4 \mathrm{ab}$ & 0.3 ef & 0.6 efg & $2.1 \mathrm{de}$ & $82.05 \mathrm{~cd}$ & $17.97 \mathrm{abc}$ & $12,397 \mathrm{cde}$ \\
\hline HM PM21 & Gaucho & $36.5 \mathrm{bcd}$ & $27.0 \mathrm{c}$ & $0.1 \mathrm{f}$ & $0.1 \mathrm{~g}$ & $1.8 \mathrm{ef}$ & $85.14 \mathrm{bc}$ & $18.12 \mathrm{ab}$ & $12,896 \mathrm{bc}$ \\
\hline HM PM21 & Poncho Beta & $37.1 \mathrm{bcd}$ & $0.5 \mathrm{e}$ & $0.2 \mathrm{f}$ & $0.1 \mathrm{~g}$ & $0.6 \mathrm{~g}$ & $85.77 \mathrm{bc}$ & $18.30 \mathrm{a}$ & $13,219 \mathrm{ab}$ \\
\hline Beta 8600 & Untreated check & $45.4 \mathrm{a}$ & $78.4 \mathrm{~b}$ & 0.4 ef & $0.9 \mathrm{e}$ & $2.8 \mathrm{~cd}$ & $88.30 \mathrm{~b}$ & $17.28 \mathrm{~d}$ & $12,671 \mathrm{bcd}$ \\
\hline Beta 8600 & Gaucho & $45.2 \mathrm{a}$ & $24.5 \mathrm{c}$ & 0.3 ef & 0.7 ef & $2.1 \mathrm{de}$ & $92.45 \mathrm{a}$ & $17.76 \mathrm{abcd}$ & $13,706 \mathrm{a}$ \\
\hline Beta 8600 & Poncho Beta & $41.5 \mathrm{ab}$ & $0.4 \mathrm{e}$ & $0.2 \mathrm{f}$ & $0.2 \mathrm{fg}$ & $1.2 \mathrm{fg}$ & $94.58 \mathrm{a}$ & $17.23 \mathrm{~d}$ & $13,338 \mathrm{ab}$ \\
\hline Phoenix R & Untreated check & 38.8 bcd & $91.5 \mathrm{a}$ & $1.9 \mathrm{~b}$ & $3.9 \mathrm{~b}$ & $4.5 \mathrm{~b}$ & $78.62 \mathrm{de}$ & $17.84 \mathrm{abcd}$ & $12,121 \mathrm{de}$ \\
\hline Phoenix R & Gaucho & $41.0 \mathrm{ab}$ & $31.8 \mathrm{c}$ & $1.1 \mathrm{~cd}$ & $3.0 \mathrm{c}$ & $4.0 \mathrm{~b}$ & $86.15 \mathrm{~b}$ & $17.28 \mathrm{~d}$ & 12,436 cde \\
\hline Phoenix R & Poncho Beta & $39.8 \mathrm{abc}$ & $0.2 \mathrm{e}$ & $0.7 \mathrm{de}$ & $1.6 \mathrm{~d}$ & $3.1 \mathrm{c}$ & $94.76 \mathrm{a}$ & $17.57 \mathrm{bcd}$ & $13,604 \mathrm{a}$ \\
\hline Monohikari & Untreated check & 34.6 cde & $80.9 \mathrm{~b}$ & $2.7 \mathrm{a}$ & $5.3 \mathrm{a}$ & $5.6 \mathrm{a}$ & $40.24 \mathrm{~g}$ & $16.54 \mathrm{e}$ & $5,658 \mathrm{~g}$ \\
\hline Monohikari & Gaucho & $33.4 \mathrm{ed}$ & $10.5 \mathrm{~d}$ & $1.3 \mathrm{c}$ & $3.8 \mathrm{~b}$ & $4.7 \mathrm{~b}$ & $58.19 \mathrm{f}$ & $17.40 \mathrm{~cd}$ & $8,799 \mathrm{f}$ \\
\hline Monohikari & Poncho Beta & $30.5 \mathrm{e}$ & $0.1 \mathrm{e}$ & $0.3 \mathrm{ef}$ & $1.0 \mathrm{e}$ & $2.1 \mathrm{de}$ & $76.96 \mathrm{e}$ & $18.19 \mathrm{ab}$ & $11,850 \mathrm{e}$ \\
\hline$P>F^{\mathrm{z}}$ & & $<0.0001$ & $<0.0001$ & $<0.0001$ & $<0.0001$ & $<0.0001$ & $<0.0001$ & $<0.0001$ & $<0.0001$ \\
\hline $\operatorname{LSD}(P \leq 0.05)$ & & 5.8 & 9.2 & 0.5 & 0.5 & 0.7 & 4.06 & 0.63 & 705 \\
\hline
\end{tabular}

u Previously established curly top resistance in hybrids: HM PM21 = high, Beta $8600=$ high-intermediate, Phoenix R = low-intermediate, and Monohikari = susceptible.

${ }^{v}$ All seed was treated with Allegence FL $(15.6 \mathrm{~g}$ a.i./100 kg) and Thiram 42S $(250 \mathrm{~g}$ a.i./100 kg). Untreated check $=$ no insecticide treatment, Gaucho $=$ imidacloprid $45 \mathrm{~g}$ a.i./100,000 seed, Poncho Beta = clothianidin $60 \mathrm{~g}$ a.i./100,000 seed + beta-cyfluthrin $8 \mathrm{~g}$ a.i./100,000 seed.

${ }^{\mathrm{w}}$ Number of plants at the cotyledon growth stage (prior to thinning) were counted in $3 \mathrm{~m}$ of row.

${ }^{x}$ Number of plants in the center two rows (total of $21.4 \mathrm{~m}$ ) with spinach leafminer (Pegomya hyoscyami) damage on 21 June.

y The curly top disease index scale ranged from $0=$ no symptoms to $9=$ dead plant (Table 1). Rating system was published by David Mumford in 1974 (11).

${ }^{\mathrm{z}} P>F$ was the probability associated with the $F$ value. LSD = Fisher's protected least significant difference value. Means followed by the same letter did not differ significantly based on Fisher's protected least significant difference value with $P \leq 0.05$. 
wide (56- $\mathrm{cm}$ row spacing) and $10 \mathrm{~m}$ long. At the two-leaf stage, the following herbicides were applied in a $22.86-\mathrm{cm}$ band: Progress $877 \mathrm{ml} / \mathrm{ha}$, Stinger $(40.9 \%$ clopyralid) $183 \mathrm{ml} / \mathrm{ha}$, Upbeet $5.8 \mathrm{~g}$ a.i./ha, and methylated seed oil $877 \mathrm{ml} / \mathrm{ha}$. At the four-leaf stage, the field was top dressed with $156 \mathrm{~kg} \mathrm{~N} / \mathrm{ha}$ impregnated with the herbicide Outlook $(63.9 \%$ dimethenamidP) at a rate of 7.6 liters per metric ton of fertilizer. The field was sprayed on 17 July with the fungicide Gem (25\% trifloxystrobin) at $420 \mathrm{~g} / \mathrm{ha}$ for powdery mildew control and the insecticide Lorsban $4 \mathrm{E}$ (44.9\% chlorpyrifos) at 1.754 liters/ha for aphid control. The crop was managed by the grower using accepted cultural practices. A stand count was taken on 15 April, prior to thinning, when the plants had only cotyledons and true leaves had not yet developed. Disease data were recorded for the center two rows on 15 July, 16 August, and 8 September using a disease index of 0 to 9 (Table 1). The center two rows were hand-harvested on 20 October with the aid of a mechanical topper and a two-row lifter. Yield data from replication 8 was not included in the analysis because of obvious field irregularities. Two eight-beet sugar samples per plot were collected for sugar analysis during harvest.

Sugar analysis. Sugar content of the beets was determined by the Amalgamated Sugar Co. laboratory using a polarimeter. Recoverable sugar was estimated based on root yield, percent sugar, and conductivity.

Data analysis. Data were analyzed in SAS (15) using the general linear models procedure, and Fisher's protected least significant difference was used for mean comparisons. Mean comparisons across treatments were conducted using single degree-of-freedom contrast statements in SAS.

\section{RESULTS}

There were no significant differences in stands (Tables 2 to 5) between insecticide treatments and untreated checks on any of the four cultivars tested based on the number of plants per row prior to thinning. Thus, a good stand was established for each cultivar at both locations and no phytotoxicity was evident.

A considerable natural leafminer infestation occurred at the Kimberly field (Table 2) but was not present at the Nampa field. The Poncho Beta treatment was significantly (Tables 2 and 4) more effective than Gaucho for leafminer control across cultivars, essentially eliminating the leafminers (0.3\% infested plants). The Gaucho treatment (23.4\% infested plants) was not as effective as Poncho Beta but was considerably better than the untreated check (83.3\% infested plants).

Moderate to severe curly top occurred at both locations and was only based on natural inoculum and natural leafhopper infestations (Tables 2 and 3). When comparing treatments, we observed significant differences in curly top ratings at both locations for all three rating periods (Tables 2 and $3)$. These differences became larger as the season progressed. PM21 was not significantly different from Beta 8600 at either location based on the July curly top reading contrasts $(P=0.4362$ and 0.3401$)$. As the season progressed, Beta 8600 developed more curly top symptoms than PM21 based on contrasts for two additional curly top readings (August $P=0.0211$ and 0.0030 and September $P=0.0073$ and $<0.0001)$. Both PM21 and Beta 8600 were more resistant to curly top than Phoenix R and Monohikari at both locations on all three rating dates based on contrasts $(P<$ 0.0001). Curly top symptom expression across treatments was significantly lower in Phoenix $\mathrm{R}$ than in Monohikari in Nampa (July $P=0.0028$, August $P<$ 0.0001 , and September $P<0.0001$ ) but varied with rating date at Kimberly (July $P$ $=0.1216$, August $P=0.0009$, and September $P=0.1756$ ). Both insecticide treatments had lower curly top ratings than the untreated checks at both locations across hybrids based on contrasts (Tables 4 and 5). The plots treated with Poncho Beta had lower curly top ratings than plots treated with Gaucho in all cases except for the first rating date in Nampa.

Based on contrasts, treatment with Poncho Beta resulted in increased yield and estimated recoverable sugar more than Gaucho, but the Gaucho seed treatment performed better than untreated seed (Tables 4 and 5). When considering the individual hybrids (Tables 2 and 3), although Poncho Beta did not always result in yield greater than Gaucho in the hybrids with better curly top resistance, there was a trend for the Poncho Beta root yield to be higher than Gaucho and the Gaucho seed treatment to perform better than the untreated seed. Comparative results demonstrated that Poncho Beta increased sugar content, but Gaucho did not. However, Poncho Beta did not always result in significantly improved sugar content on the more resistant hybrids (Tables 2 and 3).

\section{DISCUSSION}

Under moderate to heavy infection pressure, seed treatment with Poncho Beta was able to provide acceptable protection from curly top on all sugar beet hybrids tested. Even sugar beet hybrids highly susceptible

Table 3. Influence of sugar beet host resistance and insecticide seed treatments on curly top during the 2005 growing season in Nampa, ID

\begin{tabular}{|c|c|c|c|c|c|c|c|c|}
\hline \multirow{2}{*}{$\begin{array}{l}\text { Sugar beet } \\
\text { hybrid }^{v}\end{array}$} & \multirow{2}{*}{$\begin{array}{l}\text { Insecticide seed } \\
\text { treatment }^{\text {w }}\end{array}$} & \multirow{2}{*}{$\begin{array}{l}\text { No. plants } \\
\text { per } 3 \text { m }^{\mathrm{x}}\end{array}$} & \multicolumn{3}{|c|}{ Curly top ratingy } & \multirow{2}{*}{$\begin{array}{l}\text { Root } \\
\text { yield } \\
\text { (t/ha) }\end{array}$} & \multirow{2}{*}{$\begin{array}{c}\text { Sugar } \\
\text { content } \\
(\%)\end{array}$} & \multirow{2}{*}{$\begin{array}{c}\text { Estimated } \\
\text { recoverable } \\
\text { sugar } \\
(\mathrm{kg} / \mathrm{ha})\end{array}$} \\
\hline & & & $15 \mathrm{Jul}$ & 16 Aug & $8 \mathrm{Sep}$ & & & \\
\hline HM PM21 & Untreated check & $31.4 \mathrm{abcd}$ & $0.8 \mathrm{def}$ & $1.1 \mathrm{fg}$ & $2.7 \mathrm{fg}$ & $97.65 \mathrm{~cd}$ & $17.38 \mathrm{a}$ & $14,880 \mathrm{c}$ \\
\hline HM PM21 & Gaucho & $34.5 \mathrm{ab}$ & $0.4 \mathrm{ef}$ & $0.8 \mathrm{gh}$ & $2.6 \mathrm{fg}$ & $100.43 \mathrm{c}$ & $17.56 \mathrm{a}$ & $15,400 \mathrm{bc}$ \\
\hline HM PM21 & Poncho Beta & $29.4 \mathrm{~cd}$ & $0.2 \mathrm{f}$ & $0.1 \mathrm{~h}$ & $1.5 \mathrm{~h}$ & $102.09 \mathrm{bc}$ & $17.27 \mathrm{a}$ & $15,376 \mathrm{bc}$ \\
\hline Beta 8600 & Untreated check & $32.4 \mathrm{abc}$ & $0.4 \mathrm{def}$ & $2.2 \mathrm{de}$ & $3.7 \mathrm{e}$ & $111.39 \mathrm{ab}$ & $17.20 \mathrm{a}$ & $16,668 \mathrm{ab}$ \\
\hline Beta 8600 & Gaucho & $35.6 \mathrm{a}$ & $0.2 \mathrm{f}$ & 1.8 ef & $3.1 \mathrm{f}$ & $115.90 \mathrm{a}$ & $17.24 \mathrm{a}$ & $17,482 \mathrm{a}$ \\
\hline Beta 8600 & Poncho Beta & $31.0 \mathrm{bcd}$ & $0.1 \mathrm{f}$ & $0.2 \mathrm{gh}$ & $2.1 \mathrm{~g}$ & $116.52 \mathrm{a}$ & $17.16 \mathrm{ab}$ & $17,341 \mathrm{a}$ \\
\hline Phoenix R & Untreated check & $28.1 \mathrm{~cd}$ & $2.8 \mathrm{a}$ & $4.4 \mathrm{bc}$ & $5.3 \mathrm{c}$ & $82.70 \mathrm{e}$ & $16.21 \mathrm{c}$ & $11,702 \mathrm{e}$ \\
\hline Phoenix R & Gaucho & $30.6 \mathrm{bcd}$ & $1.5 \mathrm{bc}$ & $4.1 \mathrm{c}$ & $4.7 \mathrm{~d}$ & $90.21 \mathrm{de}$ & $16.13 \mathrm{c}$ & $12,632 \mathrm{de}$ \\
\hline Phoenix R & Poncho Beta & $29.6 \mathrm{~cd}$ & 0.9 cde & $2.6 \mathrm{~d}$ & $3.8 \mathrm{e}$ & $100.18 \mathrm{c}$ & $16.31 \mathrm{bc}$ & $14,032 \mathrm{~cd}$ \\
\hline Monohikari & Untreated check & $29.9 \mathrm{~cd}$ & $1.7 \mathrm{~b}$ & $6.4 \mathrm{a}$ & $7.3 \mathrm{a}$ & $44.92 \mathrm{~g}$ & $14.69 \mathrm{~d}$ & $5,898 \mathrm{~g}$ \\
\hline Monohikari & Gaucho & $27.2 \mathrm{~d}$ & $1.1 \mathrm{bcd}$ & $5.2 \mathrm{~b}$ & $6.5 \mathrm{~b}$ & $57.43 \mathrm{f}$ & $15.58 \mathrm{c}$ & $7,798 \mathrm{f}$ \\
\hline Monohikari & Poncho Beta & $31.5 \mathrm{abcd}$ & 0.6 def & $2.7 \mathrm{~d}$ & $3.9 \mathrm{e}$ & $85.77 \mathrm{e}$ & $17.33 \mathrm{a}$ & $13,016 \mathrm{de}$ \\
\hline$P>F^{\mathrm{z}}$ & & 0.0230 & $<0.0001$ & $<0.0001$ & $<0.0001$ & $<0.0001$ & $<0.0001$ & $<0.0001$ \\
\hline $\operatorname{LSD}(P \leq 0.05)$ & & 4.6 & 0.7 & 0.9 & 0.5 & 9.57 & 0.87 & 1,532 \\
\hline
\end{tabular}

${ }^{\mathrm{v}}$ Previously established curly top resistance in hybrids: HM PM21 = high, Beta $8600=$ high-intermediate, Phoenix R = low-intermediate, and Monohikari = susceptible.

${ }^{\mathrm{w}}$ All seed was treated with Allegence FL $(15.6 \mathrm{~g}$ a.i. $/ 100 \mathrm{~kg})$ and Thiram $42 \mathrm{~S}(250 \mathrm{~g}$ a.i./100 kg). Untreated check = no insecticide treatment, Gaucho = imidacloprid $45 \mathrm{~g}$ a.i./100,000 seed, Poncho Beta = clothianidin $60 \mathrm{~g}$ a.i./100,000 seed + beta-cyfluthrin $8 \mathrm{~g}$ a.i./100,000 seed.

$\mathrm{x}$ Number of plants at the cotyledon growth stage (prior to thinning) were counted in $3 \mathrm{~m}$ of row.

y The curly top disease index scale ranged from $0=$ no symptoms to $9=$ dead plant (Table 1). Rating system was published by David Mumford in 1974 (11).

${ }^{\mathrm{z}} P>F$ was the probability associated with the $F$ value. LSD = Fisher's protected least significant difference value. Means followed by the same letter did not differ significantly based on Fisher's protected least significant difference value with $P \leq 0.05$. 
Table 4. Single degree-of-freedom contrasts on data pooled across four sugar beet hybrids to investigate the influence of host resistance and insecticide seed treatments on the control of curly top during the 2005 growing season near Kimberly, ID

\begin{tabular}{|c|c|c|c|c|}
\hline Variable & Contrast (mean)w & $\begin{array}{c}\text { Percent } \\
\text { difference }\end{array}$ & $\boldsymbol{F}$ & $P>F$ \\
\hline $\operatorname{Stand}^{\mathrm{x}}$ & $\begin{array}{l}\text { Control (39.5) vs. Gaucho (39.0) } \\
\text { Control (39.5) vs. Poncho Beta (37.2) } \\
\text { Gaucho (39.0) vs. Poncho Beta (37.2) }\end{array}$ & $\begin{array}{l}-1.3 \\
-5.8 \\
-4.6\end{array}$ & $\begin{array}{l}0 \\
2 \\
2\end{array}$ & $\begin{array}{l}0.7502 \\
0.1241 \\
0.2204\end{array}$ \\
\hline Plants with leafminer ${ }^{y}$ & $\begin{array}{l}\text { Control (82.3) vs. Gaucho (23.4) } \\
\text { Control (82.3) vs. Poncho Beta (0.3) } \\
\text { Gaucho (23.4) vs. Poncho Beta (0.3) }\end{array}$ & $\begin{array}{l}-71.6 \\
-99.6 \\
-98.7\end{array}$ & $\begin{array}{r}677 \\
1301 \\
101\end{array}$ & $\begin{array}{l}<0.0001 \\
<0.0001 \\
<0.0001\end{array}$ \\
\hline Curly top rating ${ }^{z} 13$ July & $\begin{array}{l}\text { Control (1.32) vs. Gaucho (0.72) } \\
\text { Control (1.32) vs. Poncho Beta }(0.34) \\
\text { Gaucho }(0.72) \text { vs. Poncho Beta }(0.34)\end{array}$ & $\begin{array}{l}-45.4 \\
-74.2 \\
-52.8\end{array}$ & $\begin{array}{l}28 \\
73 \\
11\end{array}$ & $\begin{array}{r}<0.0001 \\
<0.0001 \\
0.0017\end{array}$ \\
\hline Curly top rating 9 Aug & $\begin{array}{l}\text { Control (2.69) vs. Gaucho (1.91) } \\
\text { Control (2.69) vs. Poncho Beta }(0.75) \\
\text { Gaucho (1.91) vs. Poncho Beta }(0.75)\end{array}$ & $\begin{array}{l}-29.0 \\
-72.1 \\
-60.7\end{array}$ & $\begin{array}{r}36 \\
221 \\
79\end{array}$ & $\begin{array}{l}<0.0001 \\
<0.0001 \\
<0.0001\end{array}$ \\
\hline Curly top rating $7 \mathrm{Sept}$ & $\begin{array}{l}\text { Control (3.74) vs. Gaucho (3.14) } \\
\text { Control (3.74) vs. Poncho Beta (1.77) } \\
\text { Gaucho (3.14) vs. Poncho Beta (1.77) }\end{array}$ & $\begin{array}{l}-16.0 \\
-52.7 \\
-43.6\end{array}$ & $\begin{array}{r}12 \\
125 \\
60\end{array}$ & $\begin{aligned} & 0.0010 \\
< & 0.0001 \\
< & 0.0001\end{aligned}$ \\
\hline Yield (t/ha) & $\begin{array}{l}\text { Control }(72.29) \text { vs. Gaucho }(80.48) \\
\text { Control }(72.29) \text { vs. Poncho Beta }(88.01) \\
\text { Gaucho }(80.48) \text { vs. Poncho Beta }(88.01)\end{array}$ & $\begin{array}{r}11.3 \\
21.7 \\
9.4\end{array}$ & $\begin{array}{r}64 \\
237 \\
55\end{array}$ & $\begin{array}{l}<0.0001 \\
<0.0001 \\
<0.0001\end{array}$ \\
\hline Sugar $(\%)$ & $\begin{array}{l}\text { Control (17.41) vs. Gaucho (17.64) } \\
\text { Control (17.41) vs. Poncho Beta (17.82) } \\
\text { Gaucho (17.64) vs. Poncho Beta (17.82) }\end{array}$ & $\begin{array}{l}1.3 \\
2.4 \\
1.0\end{array}$ & $\begin{array}{l}2 \\
7 \\
1\end{array}$ & $\begin{array}{l}0.1496 \\
0.0109 \\
0.2520\end{array}$ \\
\hline Estimated recoverable sugar (kg/ha) & $\begin{array}{l}\text { Control }(10,712) \text { vs. Gaucho }(11,959) \\
\text { Control }(10,712) \text { vs. Poncho Beta }(13,003) \\
\text { Gaucho }(11,959) \text { vs. Poncho Beta }(13,003)\end{array}$ & $\begin{array}{r}11.6 \\
21.4 \\
8.7\end{array}$ & $\begin{array}{r}50 \\
167 \\
35\end{array}$ & $\begin{array}{l}<0.0001 \\
<0.0001 \\
<0.0001\end{array}$ \\
\hline
\end{tabular}

${ }^{\mathrm{w}}$ Treatments included sugar beet hybrids previously characterized for curly top resistance: $\mathrm{HM}$ PM21 = high, Beta $8600=$ high-intermediate, Phoenix R = low-intermediate, and Monohikari = susceptible. Treatments also included various insecticide seed treatments: Control = no insecticide treatment, Gaucho = imidacloprid $45 \mathrm{~g}$ a.i./100,000 seed, Poncho Beta = clothianidin $60 \mathrm{~g}$ a.i./100,000 seed + beta-cyfluthrin $8 \mathrm{~g}$ a.i./100,000 seed. All seed was treated with Allegence FL (15.6 g a.i./100 kg) and Thiram 42S (250 g a.i./100 kg). Mean = mean value for data pooled across hybrids. Percent difference = percent difference between means for treatments in contrast.

${ }^{\mathrm{x}}$ Number of plants at the cotyledon growth stage (prior to thinning) were counted in $3 \mathrm{~m}$ of row.

${ }^{y}$ Percentage of plants in the center two rows (total of $21.4 \mathrm{~m}$ ) with spinach leafminer (Pegomya hyoscyami) on 21 June.

$\mathrm{z}$ The curly top disease index scale ranged from $0=$ no symptoms to $9=$ dead plant.

to curly top had commercially acceptable levels of curly top at the end of the season and reasonable yield parameters under moderate disease pressure. The Poncho Beta treatment outperformed both Gaucho and the untreated check in curly top ratings (except for first rating at Nampa), root yield, and estimated recoverable sugar based on contrasts. When looking at simple effects based on Fisher's protected least significant differences (LSD), Poncho Beta did not always outperform other treatments based on ratings and yield parameters. When considering root yield data, there was a trend for Poncho Beta to consistently result in higher yield than the other treatments. This trend was significant with the susceptible hybrids but not with PM21 and Beta 8600, hybrids with better host resistance. When considering estimated recoverable sugar, trends were not clear with the more resistant hybrids. This decrease in ability to detect significant differences for recoverable sugar was likely because of utilizing several parameters (root yield, sugar content, and conductivity) to estimate the values, each of which adds additional variation.

Gaucho also outperformed the untreated check by reducing foliar curly top symptoms and increasing root yield and estimated recoverable sugar based on con- trasts. When comparing simple effects with Fisher's protected LSD, trends and significant differences were not always present. Differences were more common with parameters associated with the more susceptible hybrids. The limited level of protection provided by imidacloprid in this study is consistent with previous research (8) where this product was unable to prevent large yield losses among susceptible hybrids. The previous work also established that imidacloprid and phorate provided similar levels of protection to curly top on sugar beets.

The clothianidin in Poncho and imidacloprid in Gaucho are both in the neonicotinoid chemical family. This chemical family specifically targets nicotinic acetylcholine receptors leading to death of the insect (13). This selectivity for insects means they have a very low mammalian toxicity, and they do not accumulate in mammals or through food chains $(13,20)$. Imidacloprid, released in 1991, was the first neonicotinoid on the market. Second generation neonicotinoids such as clothianidin, thiamethoxam, dinotefuran, and acetamiprid are now becoming available. With the speed and scale with which imidacloprid was released and incorporated into control strategies around the world, there was concern about develop- ment of resistant insect populations (13). To a large extent, these worries have not been realized in practice, and imidacloprid has proven remarkably resilient (13). Extensive surveys have shown neonicotinoid resistance remains restricted to few insect species and often very localized (13). As additional neonicotinoids continue to be commercialized, this class of synthetic insecticides will require continued monitoring for the development of insect resistance (20).

Host resistance remains the most economical and primary defense against curly top in sugar beets. However, host resistance is not complete and is inherited in a quantitative manner $(2,8)$. As the sugar beet industry readily incorporates resistance to other diseases such as rhizomania into new hybrids, developing high levels of the quantitatively inherited curly top resistance in all parental lines used in hybrid development cannot be easily or rapidly achieved. In addition, after adding multiple sources of disease resistance, maintaining yield potential is difficult. To aid in curly top control in California, a curly top management program has been in place since 1943 (21). This program is based in part on the migratory behavior of $C$. tenellus and employs aerial insecticide applications directed toward noncrop areas where the 
Table 5. Single degree-of-freedom contrasts on data pooled across four sugar beet hybrids to investigate the influence of host resistance and insecticide seed treatments on the control of curly top during the 2005 growing season near Nampa, ID

\begin{tabular}{|c|c|c|c|c|}
\hline Variable & Contrast (mean) $)^{x}$ & $\begin{array}{c}\text { Percent } \\
\text { difference }\end{array}$ & $\boldsymbol{F}$ & $P>F$ \\
\hline Stand ${ }^{y}$ & $\begin{array}{l}\text { Control (30.4) vs. Gaucho (32.0) } \\
\text { Control (30.4) vs. Poncho Beta (30.4) } \\
\text { Gaucho (32.0) vs. Poncho Beta (30.4) }\end{array}$ & $\begin{array}{r}5.3 \\
0.0 \\
-5.0\end{array}$ & $\begin{array}{l}2 \\
0 \\
2\end{array}$ & $\begin{array}{l}0.1791 \\
0.9569 \\
0.1626\end{array}$ \\
\hline Curly top rating ${ }^{z} 15$ July & $\begin{array}{l}\text { Control (1.41) vs. Gaucho }(0.80) \\
\text { Control }(1.41) \text { vs. Poncho Beta }(0.48) \\
\text { Gaucho }(0.80) \text { vs. Poncho Beta }(0.48)\end{array}$ & $\begin{array}{l}-43.3 \\
-66.0 \\
-40.0\end{array}$ & $\begin{array}{r}13 \\
30 \\
3\end{array}$ & $\begin{array}{r}0.0006 \\
<0.0001 \\
0.0685\end{array}$ \\
\hline Curly top rating 16 Aug & $\begin{array}{l}\text { Control (3.53) vs. Gaucho (2.95) } \\
\text { Control (3.53) vs. Poncho Beta (1.42) } \\
\text { Gaucho (2.95) vs. Poncho Beta (1.42) }\end{array}$ & $\begin{array}{l}-16.4 \\
-59.8 \\
-51.9\end{array}$ & $\begin{array}{r}7 \\
94 \\
49\end{array}$ & $\begin{array}{r}0.0096 \\
<0.0001 \\
<0.0001\end{array}$ \\
\hline Curly top rating $8 \mathrm{Sept}$ & $\begin{array}{l}\text { Control (4.74) vs. Gaucho (4.22) } \\
\text { Control (4.74) vs. Poncho Beta (2.84) } \\
\text { Gaucho (4.22) vs. Poncho Beta (2.84) }\end{array}$ & $\begin{array}{l}-11.0 \\
-40.1 \\
-32.7\end{array}$ & $\begin{array}{r}14 \\
194 \\
103\end{array}$ & $\begin{array}{r}0.0003 \\
<0.0001 \\
<0.0001\end{array}$ \\
\hline Yield (t/ha) & $\begin{array}{l}\text { Control }(84.15) \text { vs. Gaucho }(90.99) \\
\text { Control }(84.15) \text { vs. Poncho Beta }(101.15) \\
\text { Gaucho }(90.99) \text { vs. Poncho Beta }(101.15)\end{array}$ & $\begin{array}{r}8.1 \\
20.2 \\
11.2\end{array}$ & $\begin{array}{r}8 \\
50 \\
18\end{array}$ & $\begin{array}{r}0.0058 \\
<0.0001 \\
<0.0001\end{array}$ \\
\hline Sugar $(\%)$ & $\begin{array}{l}\text { Control (16.37) vs. Gaucho (16.63) } \\
\text { Control (16.37) vs. Poncho Beta (17.02) } \\
\text { Gaucho (16.63) vs. Poncho Beta (17.02) }\end{array}$ & $\begin{array}{l}1.6 \\
4.0 \\
2.3\end{array}$ & $\begin{array}{l}1 \\
9 \\
3\end{array}$ & $\begin{array}{l}0.2420 \\
0.0039 \\
0.0746\end{array}$ \\
\hline Estimated recoverable sugar $(\mathrm{kg} / \mathrm{ha})$ & $\begin{array}{l}\text { Control }(12,287) \text { vs. Gaucho }(13,328) \\
\text { Control }(12,287) \text { vs. Poncho Beta }(14,941) \\
\text { Gaucho }(13,328) \text { vs. Poncho Beta }(14,941)\end{array}$ & $\begin{array}{r}8.5 \\
21.6 \\
12.1\end{array}$ & $\begin{array}{r}7 \\
48 \\
18\end{array}$ & $\begin{array}{r}0.0085 \\
<0.0001 \\
<0.0001\end{array}$ \\
\hline
\end{tabular}

${ }^{\mathrm{x}}$ Treatments included sugar beet hybrids previously characterized for curly top resistance: HM PM21 = high, Beta $8600=$ high-intermediate, Phoenix R $=$ low-intermediate, and Monohikari $=$ susceptible. Treatments also included various insecticide seed treatments: Control $=$ no insecticide treatment, Gaucho 600 = imidacloprid $45 \mathrm{~g}$ a.i./100,000 seed, Poncho Beta = clothianidin $60 \mathrm{~g}$ a.i./100,000 seed + beta-cyfluthrin $8 \mathrm{~g}$ a.i./100,000 seed. All seed was treated with Allegence FL (15.6 g a.i./100 kg) and Thiram 42S (250 g a.i./100 kg). Mean = mean value for data pooled across hybrids. Percent difference = percent difference between means for treatments in contrast.

y Number of plants at the cotyledon growth stage (prior to thinning) were counted in $3 \mathrm{~m}$ of row.

${ }^{\mathrm{z}}$ Curly top disease index scale ranged from $0=$ no symptoms to $9=$ dead plant.

insect vector tends to congregate. However, as increasing urbanization occurs near farming regions, many areas are no longer accessible to spraying and nontarget organisms influenced by the sprays make aspects of this program undesirable (21). A similar curly top management program was in place in southern Idaho from 1949 through 1969 but fell out of favor because of increasing urbanization and the lack of funds (3). Adjusting sugar beet planting dates can provide some control of curly top but has proven to be inconsistent $(7,21)$. In the past, a number of insecticides (pyrethrum, DDT, malathion, phorate, disulfoton, dimethoate, aldicarb, and carbofuran) have been investigated for the control of curly top $(3,8,10,12,14)$. Each proved to be effective in killing leafhoppers but for various reasons (such as too short an efficacy period) failed to provide adequate levels of curly top control. The use of the neonicotinoids was first investigated with imidacloprid and was found to be somewhat effective but was unable to prevent large yield losses in susceptible cultivars $(8,21)$. Recently, a second generation of neonicotinoids has started to become available which includes clothianidin, the active ingredient in Poncho. Our results indicate that Poncho Beta, a combination of clothianidin $(60 \mathrm{~g}$ a.i./100,000 seed) and beta-cyfluthrin ( $8 \mathrm{~g}$ a.i./100,000 seed) was effective in reducing large losses from curly top even in very susceptible hybrids. We speculate that beta-cyfluthrin, a nonsystemic insecticide, had very little influence on the control of curly top, but the studies presented herein did not address the influence of beta-cyfluthrin. Nevertheless, the data presented indicate that second generation neonicotinoids may provide a valuable tool for reducing the incidence and severity of curly top in sugar beets in Idaho and other regions with a similar growing season. Since this disease problem is impacted by the influence of weather on leafhopper numbers and movement and disease severity, the weed population harboring both vector and virus, and hybrids varying for resistance, curly top requires multifaceted management. To enhance our disease management options, we should continue to improve on host resistance to curly top along with investigating the potential for utilizing seed treatments to supplement host resistance.

\section{ACKNOWLEDGMENTS}

These data support the objectives for the United States Department of Agriculture CRIS project 5368-21220-001-00D. We acknowledge Betaseed Inc., Amalgamated Sugar Co., Bayer CropScience, and the Snake River Sugar Beet Growers for supporting our efforts.

\section{LITERATURE CITED}

1. Baliji, S., Black, M. C., French, R., Stenger, D. C., and Sunter, G. 2004. Spinach curly top virus: A newly described Curtovirus species from southwest Texas with incongruent gene phylogenies. Phytopathology 94:772-779.

2. Bennett, C. W. 1971. The curly top disease of sugarbeet and other pests. Monogr. No. 7. American Phytopathological Society, St. Paul, MN.

3. Blickenstaff, C. C., and Traveller, D. 1979. Factors affecting curly top damage to sugarbeets and beans in southern Idaho, 191977. Science and Education Administration, Agricultural Reviews and Manuals, Western Series, No. 8. U.S. Dep. Agric.-Agric. Res. Serv., Oakland, CA.

4. Camp, S., Foote, P., Strausbaugh, C. A., and Gillen, A. M. 2005. Evaluation of commercial sugarbeet hybrids for resistance to beet curly top in Canyon County, ID, 2004. Biol. Cultural Tests Control Plant Dis. Report 20: FC023. Online publication. DOI: 10.1094/BC20. American Phytopathological Society, St. Paul, $\mathrm{MN}$.

5. Creamer, R., Hubble, H., and Lewis, A. 2005. Curtovirus infection of chile pepper in New Mexico. Plant Dis. 89:480-486.

6. Creamer, R., Luque-Williams, M., and Howo, M. 1996. Epidemiology and incidence of beet curly top geminivirus in naturally infected weed hosts. Plant Dis. 80:533-535.

7. Duffus, J. E., and Skoyen, I. O. 1977. Relationship of age of plants and resistance to a severe isolate of the beet curly top virus. Phytopathology 67:151-154.

8. Kaffka, S. R., Wintermantel, W. M., and Lewellen, R. T. 2002. Comparisons of soil and seed applied systemic insecticides to control Beet curly top virus in the San Joaquin Valley. J. Sugar Beet Res. 39:59-74.

9. Liu, H.-Y., Sears, J. L., and Lewellen, R. T. 2005. Occurrence of resistance-breaking Beet necrotic yellow vein virus of sugar beet. Plant Dis. 89:464-468.

10. Malm, N. R., and Finkner, R. E. 1968. The use of systemic insecticides to reduce the incidence of curly top virus disease in sugarbeets. J. Am. Soc. Sugar Beet Tech. 15:246-254.

11. Mumford, D. L. 1974. Procedure for inducing 
curly top epidemics in field plots. J. Am. Soc. Sugar Beet Tech. 18:20-23.

12. Mumford, D. L., and Griffin, G. D. 1973. Evaluation of systemic pesticides in controlling sugarbeet leafhopper. J. Am. Soc. Sugar Beet Tech. 17:354-357.

13. Nauen, R., and Denholm, I. 2005. Resistance of insect pests to neonicotinoid insecticides: Current status and future prospects. Arch. Insect Biochem. Physiol. 58:200-215.

14. Ritenour, G., Hills, F. J., and Lange, W. H. 1970. Effect of planting date and vector control on the suppression of curly top and yellows in sugarbeet. J. Am. Soc. Sugar Beet Tech. 16:78-84.

15. SAS Institute 1999. The SAS system for Windows. Version 8.2. SAS Institute Inc., Cary, NC.
16. Soto, M. J., and Gilbertson, R. L. 2003. Distribution and rate of movement of the curtovirus Beet mild curly top virus (family Geminiviridae) in the beet leafhopper. Phytopathology 93:478-484.

17. Stenger, D. C. 1998. Replication specificity elements of the Worland strain of beet curly top virus are compatible with those of the $\mathrm{CFH}$ strain but not those of the Cal/Logan strain. Phytopathology 88:1174-1178.

18. Stenger, D. C., and McMahon, C. L. 1997. Genotypic diversity of beet curly top virus populations in the western United States. Phytopathology 87:737-744.

19. Strausbaugh, C. A., Gillen, A. M., Camp, S., and Foote, P. 2005. Evaluation of experimental sugarbeet hybrids for resistance to beet curly top in Canyon County, ID, 2004. Biol. Cultural Tests Control Plant Dis. Report 20: FC015. Online publication. DOI: 10.1094/BC20. American Phytopathological Society, St. Paul, MN.

20. Tomizawa, M., and Casida, J. E. 2003. Selective toxicity of neonicotinoids attributable to specificity of insect and mammalian nicotinic receptors. Annu. Rev. Entomol. 48:339-364.

21. Wang, H., Gurusinghe, P. de A., and Falk, B. W. 1999. Systemic insecticides and plant age affect beet curly top virus transmission to selected host plants. Plant Dis. 83:351-355.

22. Wintermantel, W. M., and Kaffka, S. R. 2006 Sugar beet performance with curly top is related to virus accumulation and age at infection. Plant Dis. 90:657-662. 